\title{
A Complete Pathological Response to Pembrolizumab following ex vivo Liver Resection in a Patient with Colorectal Liver Metastases
}

\author{
Maria Baimas-George $^{\mathrm{a}} \quad$ Erin Baker $^{\mathrm{b}}$ Michal Kamionek $^{\mathrm{c}} \quad$ J. Stuart Salmon ${ }^{\mathrm{d}}$ \\ Amit Sastry $^{b}$ David Levi $^{\mathrm{e}}$ Dionisios Vrochides ${ }^{\mathrm{b}}$ \\ a Department of General Surgery, Carolinas Medical Center, Carolinas Healthcare Systems, Charlotte, NC, USA; \\ ${ }^{b}$ Department of Hepatopancreatobiliary Surgery, Carolinas Medical Center, Carolinas Healthcare Systems, Charlotte, \\ NC, USA; ' Department of Pathology, Carolinas Medical Center, Carolinas Healthcare Systems, Charlotte, NC, USA; \\ ${ }^{\mathrm{d}}$ Department of Medical Oncology, Carolinas Medical Center, Carolinas Healthcare Systems, Charlotte, NC, USA; \\ eDepartment of Transplant Surgery, Carolinas Medical Center, Carolinas Healthcare Systems, Charlotte, NC, USA
}

\section{Established Facts}

- Survival has improved in stage IV colorectal cancer.

- Advances in surgical technique have been made allowing R0 resection in previously considered unresectable cases.

- Rates of complete pathological responses are low.

- Pembrolizumab has shown utility in mismatch-repair-deficient colorectal carcinoma.

\section{Novel Insights}

- Treatment with pembrolizumab in our patient with Lynch syndrome led to a dramatic improvement in clinical performance and a decrease in tumor burden.

- There is treatment potential of pembrolizumab in microsatellite instability-high colorectal cancer.

- Pembrolizumab can create a chance at resectability in appropriate patients, improve survival and functional status, and may even lead to a complete pathological response in a bulky cancer.

\section{Keywords}

Cancer immunotherapy · Chemotherapy · Colorectal cancer · Hepatic metastases · Pathologically complete response Pembrolizumab

\begin{abstract}
Advances in the systemic treatment of stage IV colorectal cancer with liver metastases has offered improved survival rates for patients who otherwise face a dismal prognosis. However, a pathologically complete response (PCR) to chemotherapy for colorectal liver metastases is still rare, and its
\end{abstract}

\section{KARGER}

(C) 2018 S. Karger AG, Basel

E-Mail karger@karger.com

www.karger.com/che
Dionisios Vrochides, $\mathrm{MD}, \mathrm{PhD}$

Department of Hepatopancreatobiliary Surgery

Carolinas Medical Center, 1000 Blythe Blvd

Charlotte, NC 28204 (USA)

E-Mail Dionisios.vrochides@ carolinashealthcare.org 
significance is not fully understood. In this case report, we describe a patient who achieved PCR after neoadjuvant immunotherapy with pembrolizumab and a left hepatectomy using an ex vivo resection technique. @ 2018 S. Karger AG, Basel

\section{Introduction}

Systemic chemotherapy for metastatic colorectal cancer (mCRC) has seen significant advances. The current literature demonstrates that it can improve overall survival and resectability of liver metastases [1-4]. Furthermore, there have been great advances in surgical technique, often resulting in an $\mathrm{R} 0$ resection in cases that formerly were considered unresectable $[5,6]$. Despite these advantages, a pathologically complete response (PCR) to chemotherapy only occurs in $4-6 \%$ of cases and its impact on survival is not well established $[7,8]$. Previously, tumors involving the major vasculature at the hepatocaval confluence or the inferior vena cava (IVC) were considered unresectable, and patients faced an expected median survival of less than 1 year [7]. Over the last 2 decades, liver transplantation techniques have been implemented in other forms of liver resection [9]. First described in the 1980s, ex vivo liver resection, venovenous bypass, and total hepatic vascular exclusion are all techniques that have crossed subspecialty lines, creating opportunities rendering previously inoperable tumors operable $[9,10]$. This report describes a patient who had PCR after induction immunotherapy with pembrolizumab followed by an R0 left hepatectomy with an ex vivo technique.

\section{Case Report}

A 41-year-old woman with Lynch syndrome who presented with an obstructing and metastatic rectosigmoid colon cancer underwent low anterior resection, end colostomy, and liver biopsy, confirming a pT3N2M1 disease. Pathological analysis demonstrated a deficiency in MSH6 mismatch repair enzyme expressed by immunohistochemistry, and molecular studies identified a KRAS A146T mutation in the tumor specimen. She later underwent genetic testing, identifying the presence of a germline MSH6 mutation (3840del7), confirming Lynch syndrome. Cross-sectional imaging at diagnosis demonstrated a large unresectable central hepatic lesion measuring $11.3 \times 7.9 \mathrm{~cm}$ located mainly in segment 1 (Fig. 1a). The patient received 14 doses of palliative oxaliplatin and fluorouracil-based cytotoxic chemotherapy (mFOLFOX6) with a radiographic response, and was then transitioned to irinotecan-fluorouracil therapy (FOLFIRI) after developing chemotherapy-induced neurotoxicity. Initial early treatment with beva-
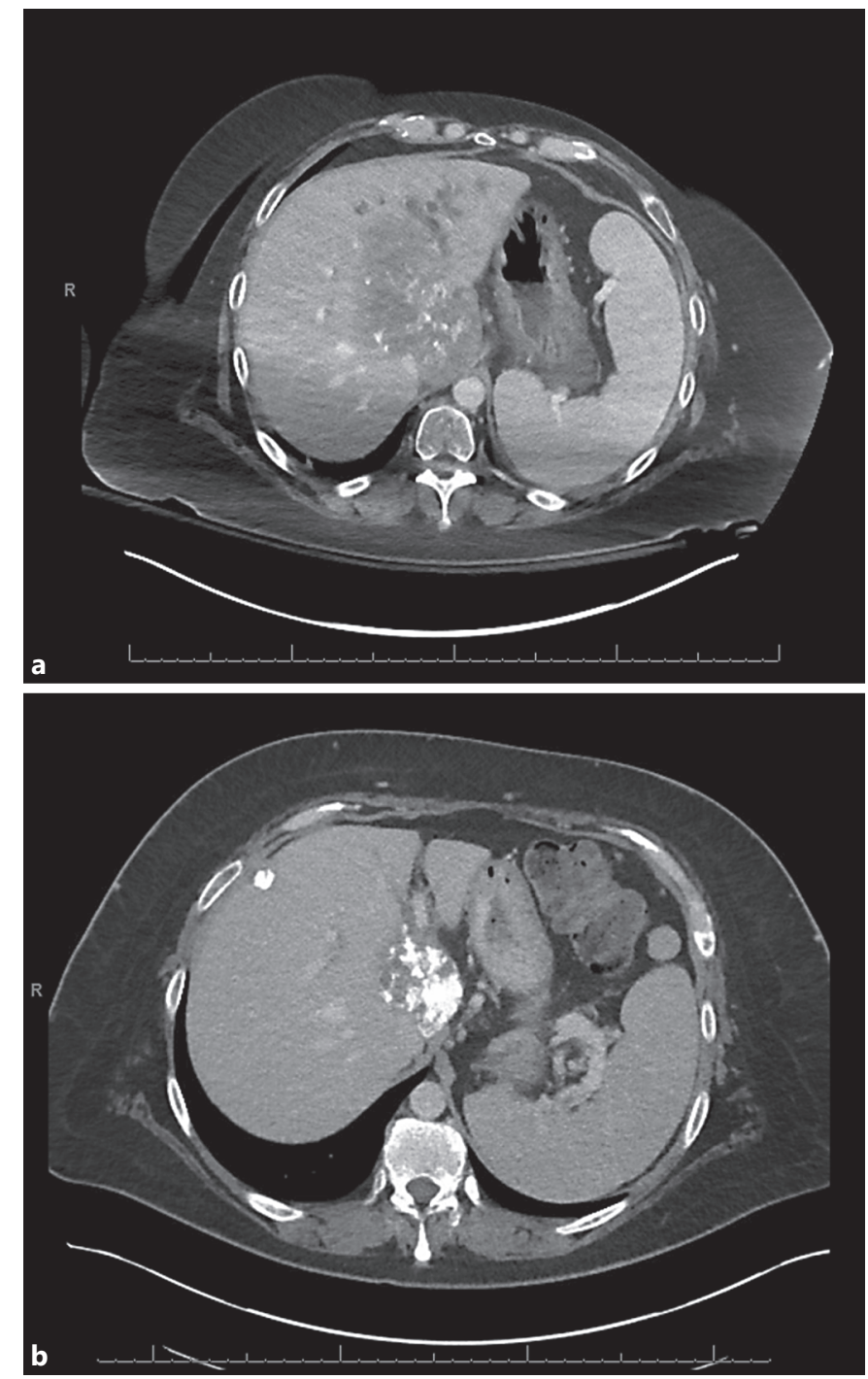

Fig. 1. CT scan images. a December 20, 2016: initial tumor diagnosis. Large central hepatic mass measuring $11 \times 9 \mathrm{~cm}$. b September 5, 2017: reduced disease burden; tumor dimensions $4.9 \times 6.7$ $\mathrm{mm}$.

cizumab was discontinued after a surgical wound dehiscence. Subsequent imaging demonstrated an increase in lesion size and 2 new smaller lesions in the right and left hepatic lobes. Furthermore, KRAS mutation was detected, and thus this therapy was discontinued. FOLFOXIRI-bevacizumab treatment was initiated but quickly discontinued after 2 months due to continued advancement of progressive disease. Pembrolizumab, a novel programmed cell death-1 (PD-1) immune checkpoint inhibitor (with recent FDA approval for this indication in May 2017), was then initiated in the setting of her microsatellite instability-high (MSI-H) tumor from Lynch syndrome. After 12 doses of pembrolizumab, the patient had demonstrated a reduction in carcinoembryonic antigen level (from 300 to $7.6 \mathrm{ng} / \mathrm{mL}$ ), radiographically decreased size of the 

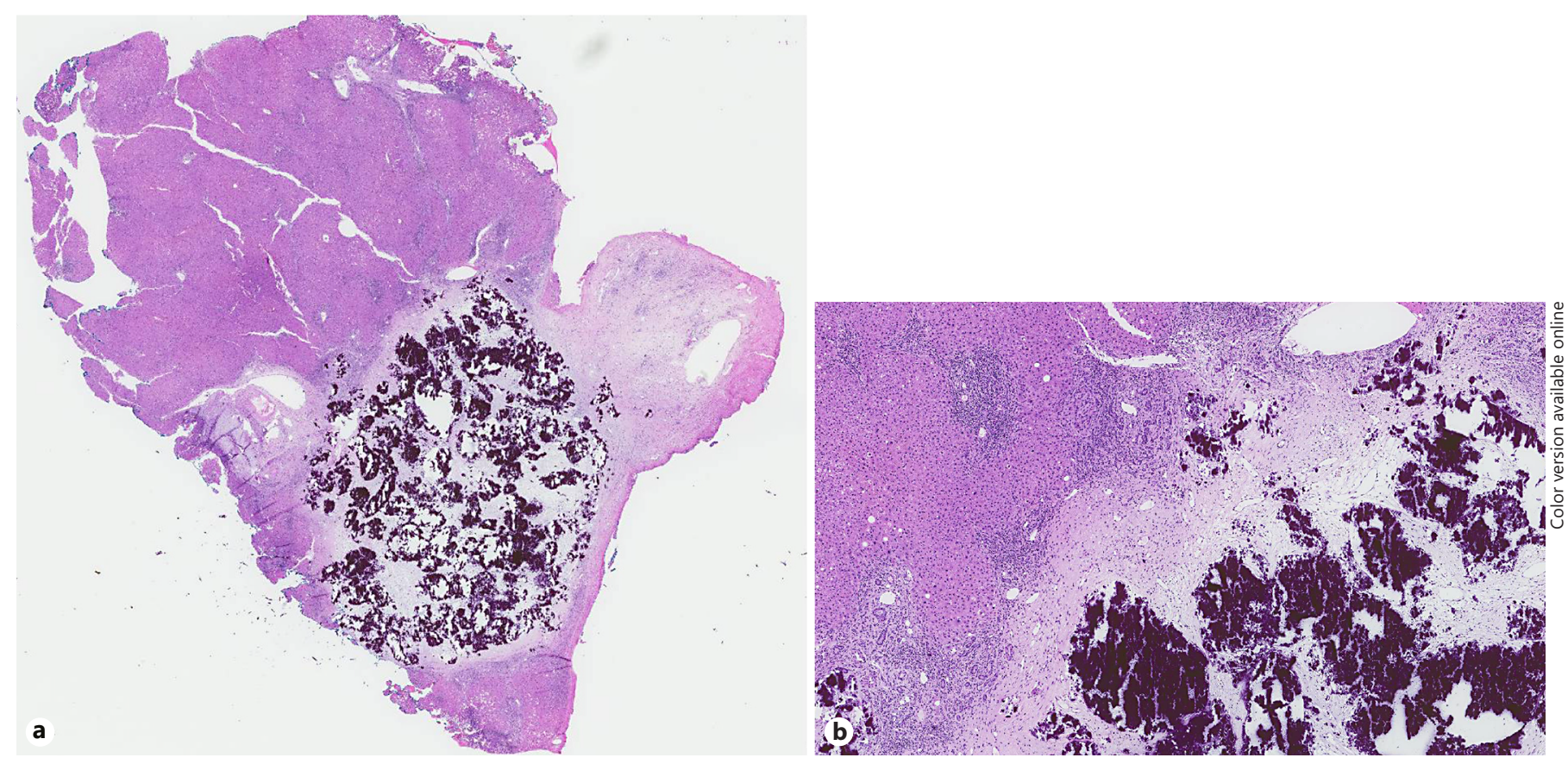

Fig. 2. a Portions of specimen demonstrating liver tissue with adjacent fibrosis and dystrophic calcification; no evidence of viable tumor. b High-power view demonstrating no viable tumor.

central hepatic lesion to $6.7 \times 4.9 \mathrm{~cm}$, and stabilization of the other 2 lesions (Fig. 1b). Given the dramatic response to systemic therapy, surgical resection was then offered. Due to the tumor's involvement of the hepatic vein and IVC confluence, the plan was to perform an ex vivo left hepatectomy, retrohepatic IVC replacement, and autotransplantation of the residual liver.

In the operating room, a large atrophic mass was identified grossly emanating from the caudate lobe extending into the left hepatic lobe. Two additional metastases previously seen on imaging were noted on the right anterior liver with no evidence of peritoneal disease. As the tumor involved the takeoff of the hepatic outflow and retrohepatic vena cava, an ex vivo resection was performed. The liver was removed en bloc with the tumor and retrohepatic IVC. The liver was cooled and flushed with preservation solution, and the resection was performed at the back table. A venovenous bypass was utilized while the patient was anhepatic, and the retrohepatic IVC was replaced with a prosthetic vascular graft. The right hemiliver was reimplanted into the abdomen. Specifically, the right hepatic vein was implanted directly into the new caval graft, and the hepatic arterial and portal venous anastomoses were performed in standard end-to-end fashion. An end-to-side Roux-en-Y hepaticojejunostomy was performed for biliary reconstruction. Lastly, 2 wedge metastectotomies were performed on the segment 6 and 7 lesions.

\section{Pathological Findings}

Pathology revealed a 6.9-cm left liver lesion with resected vena cava, consisting of pools of mucin, calcification and necrosis, but no evidence of viable tumor. The 2 smaller nodules present in the right lobe, segment 6 measuring $1.1 \times 1.0 \times 0.8$, and segment 8 measuring $2.4 \times 1.5 \times 1.0 \mathrm{~cm}$, were also found to have significant fibrosis and calcification with no evidence of viable tumor. All the caval margins were negative for viable tumor, and the 14 lymph nodes resected were also negative for evidence of carcinoma (Fig. 2a, b).

\section{Discussion}

In this report, we present a unique case of a patient with colorectal liver metastases who underwent a left hepatectomy via an ex vivo liver technique and who had a complete response to pembrolizumab following failure of traditional chemotherapy. There have been substantial advances in systemic chemotherapy for the treatment of colorectal liver metastases with studies demonstrating that neoadjuvant chemotherapy improves survival and resectability [1-4]. However, it is unclear whether a PCR plays an important prognostic role in this disease as the current literature demonstrates discordant results $[5,6]$. Blazer et al. [11] did not find a PCR to be an independent predictor of survival, although having less than $50 \%$ of residual cancer cells remaining was significantly associated. Multiple other studies have demonstrated an improvement in overall survival in complete responders 
with an overall 5-year survival rate of $76 \%$ in complete responders versus $45 \%$ without $[6,12]$. Further, the literature shows that neoadjuvant chemotherapy and negative margins of resection are important prognostic factors for survival rates and recurrence [13-15]. There is a decreased rate of additional intrahepatic recurrences, need for further resections, and improved overall survival in an $\mathrm{R} 0$ resection versus an $\mathrm{R} 1$ resection.

Pembrolizumab is a humanized monoclonal anti PD-1 antibody which blocks the negative feedback pathway of PD-1, a cell surface receptor that is involved in downregulating the immune system and suppressing the inflammatory response of T cells. Anti-PD-1 therapy thus functions to reverse the immune suppression stimulated by neoplastic cells. It has shown clinical utility in melanoma, lung cancer, and renal cell carcinoma among other cancers and has recently been approved by the FDA for use in metastatic previously treated colorectal carcinoma for patients with DNA mismatch repair enzyme deficiency and MSI-H tumors [16]. In 2015, Le et al. [17] investigated PD-1 blockade in metastatic colorectal carcinoma and found that patients with mismatch-repair-deficient colorectal carcinoma were more responsive to pembrolizumab and had an increased progression-free survival versus mismatch-repair-proficient patients. Forty percent of mismatch-repair-deficient patients had a partial response, and $50 \%$ demonstrated stable disease by RECIST criteria $[17,18]$. Several case reports have documented similar encouraging results, showing significant responses to pembrolizumab after failure of other chemotherapy regimens. A patient with a sporadic mismatchrepair-deficient mCRC with abdominal disease had shrinkage of the peritoneal tumor mass to $25 \%$ of the original tumor volume, return to normal tumor markers, and clinical performance improvement [19]. Another report of a patient with metastatic hepatocellular carcinoma who did not respond to traditional chemotherapy also had a significant improvement in tumor markers and shrinkage of tumor burden with anti-PD-1 treatment [20]. Sehdev et al. [21] described a patient who followed a very similar course as ours. The patient had MSI-H metastatic CRC with progression of disease on the following regimens: FOLFIRI-cetuximab, FOLFOX-bevacizumab, 5-fluorouracil with bevacizumab, and single-agent bevacizumab. Following treatment failure, the patient was started on pembrolizumab with dramatic improvement in clinical performance and a decrease in tumor burden after 8 weeks. After 1 year, there was no evidence of malignancy on imaging except for a residual lymph node which, after excision, was negative for viable tumor [21].
Upon a literature review, there have been only 2 other additional documented cases with anti-PD-1 therapy where the patients have achieved complete remission with pembrolizumab [22, 23].

Our patient with Lynch syndrome had a germline MSH6 gene mutation and mismatch repair enzyme deficiency, predicting benefit to treatment with anti-PD-1 therapy, and consistent with the results of the phase 2 studies of Le et al. [17]. This patient's PCR is a promising prognostic factor and demonstrates the efficacy of pembrolizumab for the treatment of $\mathrm{mCRC}$ in this population. Her pathological findings of dystrophic calcifications may also demonstrate significance in her survival and PCR. Calcifications in colorectal liver metastases can occur spontaneously prior to treatment or after systemic chemotherapy, and studies have investigated the significance of such calcifications. Easson et al. [24] demonstrated a statistically significant improvement in survival in patients with calcified metastases, independently of other variables. However, there was no correlation between complete calcification, by imaging criteria, and a PCR as viable tumor cells can persist within calcifications [25].

\section{Future Directions}

Our patient's response to pembrolizumab after failure of traditional chemotherapy adds to the existing literature, demonstrating the potential of anti-PD-1 agents in the treatment of $\mathrm{mCRC}$, and this is the first published example of the use of immunotherapy to convert previously unresectable central liver metastases to resectable disease. Even after failure of aggressive traditional chemotherapy, this case shows that pembrolizumab can create a chance at resectability in appropriate patients, improve survival and functional status, and may even lead to a PCR in a bulky cancer. Ongoing studies are testing the use of pembrolizumab as a first-line therapy in patients with MSI-H in mCRC, and future studies should continue to concentrate on drug development focused on specific molecular classifications. Additionally, although tumor calcifications appear to be a good indicator of treatment response, it may not correlate with PCR, and larger trials are needed to further assess such conclusions. An ex vivo approach to hepatic lesion resections is a complicated procedure with few case reports and series present in the literature. One such series documented a perioperative mortality rate of $33 \%$ for ex vivo resections [12]. However, it must be considered that without surgery, patients 
with central hepatic tumors face a dismal prognosis. Tumors in this location are unique because an ex vivo resection currently offers the only curative option. Patients who present with these types of tumors should be referred to tertiary care centers with experience performing such operations.

\section{Conclusion}

In this case, we highlight the extraordinary treatment potential of pembrolizumab in MSI-H CRC, and we illustrate the technically advanced operation that can be required in order to obtain an $\mathrm{R} 0$ result. This case offers improved insight into the tumor biology and treatment techniques of CRC liver metastases.

\section{Statement of Ethics}

Our research complied with the guidelines for human studies. The patient was identified by code, and the research was ethically conducted.

\section{Disclosure Statement}

The authors declare no conflicts of interest.

\section{References}

1 Nordlinger B, Sorbye H, Grimelius B, et al: Perioperative chemotherapy with FOLFOX4 and surgery versus surgery alone for resectable liver metastases from colorectal cancer (EORTC Intergroup trial 40983): a randomized controlled trial. Lancet 2008;371:10071016.

-2 Folprecht G, Grothey A, Alberts S, Raab HR, Kohne CH: Neoadjuvant treatment of unresectable colorectal liver metastases: correlation between tumour response and resection rates. Ann Oncol 2005;16:1311-1319.

-3 Van Cutsem E, Kohne CH, Lang I, et al: Cetuximab plus irinotecan, fluorouracil, and leucovorin as first-line treatment for metastatic colorectal cancer: updated analysis of overall survival according to tumor KRAS and BRAF mutation status. J Clin Oncol 2011; 29:2011-2019.

4 Van Cutsem E, Kohne CH, Hitre E, et al: Cetuximab and chemotherapy as initial treatment for metastatic colorectal cancer. N Engl J Med 2009;360:1408-1417.

5 Bengtsson G, Carlsson G, Hafstrom L, Jonsson P: Natural history of patients with untreated liver metastases from colorectal cancer. Am J Surg 1981;141:586-589.

6 Muratore A, Ribero D, Zimmitti G, Mellano A, Langella S, Capussotti L: Resection margin and recurrence-free survival after liver resection of colorectal metastases. Ann Surg Oncol 2010;17:1324-1329.

7 Adam R, Avisar E, Ariche A, et al: Five-year survival following hepatic resection after neoadjuvant therapy for nonresectable colorectal cancer. Ann Surg Oncol 2001;8:347-353.

8 Adam R, Wicherts DA, De Haas RJ, Aloia T, Levi F, Paule B, et al: Complete pathologic response after preoperative chemotherapy for colorectal liver metastases: myth or reality? J Clin Oncol 2008;26:1635-1641.

-9 Shaw BW Jr, Martin DJ, Marquez JM, et al: Venous bypass in clinical liver transplantation. Ann Surg 1984;200:524-534.
10 Yanaga K, Kishikawa K, Shimada M, et al: Extracorporeal hepatic resection for previously unresectable neoplasms. Surgery 1993;113: 637-643.

11 Blazer DG 3rd, Kishi Y, Maru DM, Kopetz S, Chun YS, Overman MJ, et al: Pathologic response to preoperative chemotherapy: a new outcome end point after resection of hepatic colorectal metastases. J Clin Oncol 2008;26: 5344-5351

12 Tanaka K, Takakura H, Takeda K, Matsuo K, Naqano Y, Endo I: Importance of complete pathologic response to prehepatectomy chemotherapy in treating colorectal cancer metastases. Ann Surg 2009;250:935-942.

13 Pawlik TM, Scoggins CR, Zorzi D, Abdalla EK, Andres A, Eng C, et al: Effect of surgical margin status on survival and site of recurrence after hepatic resection for colorectal metastases. Ann Surg 2005;241:715-722, discussion 722-724.

14 Angelsen JH, et al: Surgery for colorectal liver metastases: the impact of resection margins on recurrence and overall survival. World J Surg Oncol 2014;12:127.

15 Pandanaboyana S, White A, Pathak S, Hidalgo EL, Toogood G, Lodge JP, Prasad KR: Impact of margin status and neoadjuvant chemotherapy on survival, recurrence after liver resection for colorectal liver metastasis. Ann Surg Oncol 2015;22:173-179.

16 Brahmer JR, Tykodi SS, Chow LQ, et al: Safety and activity of anti-PD-L1 antibody in patients with advanced cancer. N Engl J Med 2012;366:2455-2465.

17 Le DT, Uram JN, Wang H, Bartlett BR, Kemberling $\mathrm{H}$, et al: $\mathrm{PD}-1$ blockade in tumors with mismatch-repair deficiency. N Engl J Med 2015;372:2509-2520.

18 Eisenhauer EA, Therasse P, Bogaerts J, Schwartz LH, Sargen D, Ford R, Dancey J, Arbuck S, Gwyther S, Mooney M, Rubinstein L, Shankar L, Dodd L, Kaplan R, Lacombe D, Verweij J: New response evaluation criteria in solid tumors: revised RECIST guideline. Eur J Cancer 2009;45:228-247.

19 Kieler M, Scheithauer W, Zielinski CC, et al: Case report: impressive response to pembrolizumab in a patient with mismatch-repair deficient metastasized colorectal cancer and bulky disease. ESMO Open 2016;1:e000084.

20 Truong P, Rahal A, Kallail K: Metastatic hepatocellular carcinoma responsive to pembrolizumab. Cureus 2016;8:e631.

21 Sehdev A, Cramer HM, Ibrahim AA, Younger AE, O'Neil BH: Pathological complete response with anti-PD-1 therapy in a patient with microsatellite instable high, BRAF mutant metastatic colon cancer: a case report and review of literature. Discov Med 2016;21: 341-347.

22 Brahmer JR, Drake CG, Wollner I, Powderly JD, Picus J, Sharfman WH, Stankevich E, Pons A, Salay TM, Mcmiller TL, Gilson MM, Wang C, Selby M, Taube JM, Anders R, Chen L, Korman AJ, Pardoll DM, Lowy I, Topalian SL: Phase I study of single-agent anti-programmed death-1 (MDX-1106) in refractory solid tumors: safety, clinical activity, pharmacodynamics, and immunologic correlates. J Clin Oncol 2010;28:31673175.

23 Lipson EJ, Sharfman WH, Drake CG, Wollner I, Taube JM, Anders RA, Xu H, Yao S, Pons A, Chen L, Pardoll DM, Brahmer JR, Topalian SL: Durable cancer regression offtreatment and effective reinduction therapy with an anti-PD-1 antibody. Clin Cancer Res 2013;19:462-468.

24 Easson AM, Barron PT, Cripps C, et al: Calcification in colorectal hepatic metastases correlates with longer survival. J Surg Oncol 1996;63:221-225.

25 Goyer P, Benoist S, Julie C, El Hajjam M, Penna $\mathrm{C}$, Nordlinger B: Complete calcification of colorectal liver metastases on imaging after chemotherapy does not indicate sterilization of disease. J Visc Surg 2012;149:271-274. 